\title{
Sensitivity of Manufacturer and Buyer's Risks for Lifetime Warranty Policies
}

\author{
Anisur Rahman ${ }^{1}$, G. N. Chattopadhyay ${ }^{2}$ \\ ${ }^{1}$ Griffith School of Engineering, Griffith University, Gold Coast, PMB 50 GCMC, QLD 9726, AUSTRALIA. \\ ${ }^{2}$ Central Queensland University, Faculty of Science, Engineering and Health Gladstone, QLD 4680, AUSTRALIA
}

\begin{abstract}
Lifetime warranties is becoming popular as they provide assurance to buyer for longer reliable service and greater customer peace of mind for the whole life of the product. By offering a lifetime warranty, both the manufacturer and the buyer are exposed to uncertainties and risks of warranty pricing and product performance during the lifetime of the product. This paper analyses the sensitivity of risk preferences models developed by Chattopadhyay and Rahman [1] in finding the optimal warranty price through the use of the manufacturer's utility function for manufacturer's profit and the buyer's utility function for repair cost. The sensitivity of the warranty price is analysed with numerical example with respect to the factors such as the buyer's and the manufacturer/dealer's risk preferences, buyer's anticipated and manufacturer's estimated product failure intensity, the buyer's loyalty to the original manufacturer/dealer in repairing failed product and the buyer's repair costs for unwarranted products.
\end{abstract}

Keywords - Lifetime warranty, risk preferences, warranty price, buyer's repair cost.

\section{INTRODUCTION}

Large numbers of products are now being sold with lifetime warranty policy since it provides assurance to buyers a long reliable service life and a better peace of mind. Under the typical situation of a lifetime warranty transaction, a buyer of a product pays for the warranty at the time of product purchase whereas, manufacturer/dealer provides rectification service in case of product failures due to design, manufacturing and quality assurance problems during the defined lifetime of the product. Under such situation, both the manufacturer and the buyer are exposed to uncertainties and risks of warranty pricing and product performance during the lifetime of the product.

By offering a lifetime warranty for a product, the manufacturer is risking in warranty pricing that whether its offer for such warranty will be accepted by the buyers. At the same time, buyers are unsure about the benefits of buying products sold with such warranty policy. Anticipation of higher product failures encourages a buyer to pay for higher warranty price which in turn encourages the manufacturer to charge a higher warranty price. In case of product covered with warranty, the buyer returns to the original manufacturer for rectification of product failures. But when the product is not covered by warranty, the buyer may take it to other service providers for rectifications. If the original manufacturer is the only one that can repair the product because of some technological monopoly, the manufacturer could charge a higher price for services and more buyers would be interested to have warranty cover. If buyers pay higher repair price for any product failures, they might be interested to buy warranty cover and even pay a higher warranty price for free rectifications. This may encourage the manufacturer to lower the warranty price to attract more buyers and to compete effectively in the market. Therefore, there is a need to model optimal warranty price which includes both buyers and manufacturers risk preferences.

Although there is a long and rich history of research efforts devoted to determining the optimal warranty price, limited works so far takes into account the manufacturer and buyer's risk preferences toward how much a manufacturer should charge and how much a buyer is willing to pay for this service. Ritchken and Tapiero [2] proposed a framework in which warranty policies for nonrepairable items can be evaluated according to risk preferences of both the manufacturer/dealer and the buyers. They emphasized the design and pricing of warranties to which the manufacturers are indifferent in an expected utility sense. Given the price warranty schedule, a buyer's response is expressed by selecting the price-warranty which minimizes disutility. As result a manufacturer can increase profit by tailoring pricewarranty schedules to specify buyer's need. Menezes [3] developed a conceptual framework to examine the impact of warranties on consumers preferences. Based on the framework, predictions of consumer preferences between product with and without a warranty were derived for various consumer segments.

Chun and Tang [4] proposed a warranty model for the free-replacement, fixed-period warranty policy that determines the optimal warranty price for ordinary base warranty. They considered a constant failure rate for the product. In line with Chun and Tang [4], Chattopadhyay and Rahman [1] proposed the warranty price models that maximizes the manufacturer/dealers certainty equivalent by applying Non-homogeneous Poisson process (NHPP) for products with time dependent failure mode. This paper analyses the sensitivity of risk preferences models developed by Chattopadhyay and Rahman [1] in finding the optimal warranty price through the use of the manufacturer's utility function for manufacturer's profit and the buyer's utility function for rectification cost. The sensitivity of the models is analyzed with numerical examples with respect to the factors such as the buyer's 
and the manufacturer's risk preferences, buyer's anticipated and manufacturer's estimated product failure intensity, the buyer's loyalty to the original manufacturer in repairing failed product and the buyer's repair costs for unwarranted products.

The outline of this paper is as follows. Section 1 briefly introduces manufacturers' and buyers' risk preferences towards a lifetime warranty policy. Section 2 provides brief overview of the Chattopadhyay and Rahman [1] risk models for lifetime warranty. Sensitivity analyses of these models are carried out in section 3 to see the effect of various factors on the warranty price from both buyer's and manufacturer's point of view. Finally, in the concluding section contribution of this research work and some recommendation of future research scopes are discussed.

\section{OVERVIEW OF CHATTOPADHYAY AND RAHMAN RISK PREFERENCE}

Because of high popularity and huge competition, manufacturers are becoming more and more interested in offering lifetime warranty for their products. Detail discussions can be found in [5]. Lifetime warranty means the manufacturer commitment to provide free or cost sharing rectification of the sold product in case of failure due to design, manufacturing or quality problems throughout the useful life of the product or the buyer's ownership period of the product. One important difference between base warranty and lifetime warranty is the coverage period. In case of base warranty it is fixed whereas it is uncertain for lifetime warranty and is often difficult to tell about life measures for the period of coverage Magnuson-Moss[6]. Termination of such warranty may arise from the technical life, technological obsolescence, commercial life/ economic life, Ownership change, social and legal life. Details can be found in Rahman and Chattopadhyay [7]. Since both manufacturer and buyer are exposed to uncertainties and risks of such warranty pricing and product performance during the lifetime of the product it is essential to develop risk preference models for both manufacture and buyer. To meet this problem Chattopadhyay and Rahman [1] developed risk preference models for lifetime warranty in finding the optimal warranty price through the use of the manufacturer's utility function for manufacturer's profit and the buyer's utility function for repair cost. The following paragraphs present and discuss the risk models developed in [1].

1) Buyer's Acceptances of lifetime Warranty model: In determining the worthy of buying a such warranty, a buyer may first estimate the total repair cost of his or her product during the defined lifetime and then compare it with the given warranty price $W$ in terms of the expected utility. Since buyer's per occasion repair $r_{b}$ is constant, a buyer's total repair cost is given by $r_{b} N_{\mathrm{b}}(L)$, ( where, $N(L)$ is the expected number of failures over the lifetime) which is estimated by his or her perceived product failure intensity $\Lambda_{b}(t)$. The higher the buyer estimates the product failure rate, the more likely he or she would be willing to buy the warranty. Considering risk averse buyer with exponential utility functions the buyer's expected warranty price for a product with time dependent failure mode is given [1] by

$W=\ln \sum_{n_{b}{ }^{*}=0}^{\infty} \exp \left(c r_{b} n_{b}{ }^{*}\right) \times \frac{\left[\left(\lambda_{b} L\right)^{\beta_{b}}\right]^{n_{b}{ }^{*}} \exp \left[-\left(\lambda_{b} L\right)^{\beta_{b}}\right]}{n_{b}{ }^{*}}$

where, $n_{b}{ }^{*}$ is the number of product failure when buyers are indifferent between the warranty price $W$ and the total repair cost $r_{b} N_{b}(L)$ in terms of the expected utility. $L$ represents the lifetime of the product. $\beta_{\mathrm{b}}$ and $\lambda_{b}$ are the shape parameter and inverse characteristic life parameter for Non-homogeneous Poisson process for individual buyer and $c$ is the risk parameters representing the buyers' risk preferences.

2) Manufacturer's profit: Similarly, considering risk averse buyer with exponential utility functions manufacturer's optimal warranty price is given [1] by

$$
\begin{aligned}
& W=-\frac{1}{a p S} \times \ln \sum_{n_{m}=0}^{\infty} e^{-a d S k n_{m}} \cdot \frac{\left[\left(\lambda_{m} L\right)^{\beta_{m}}\right] e^{-\left(\lambda_{m} L\right)^{\beta_{m}}}}{n_{m} !} \\
& +\frac{q}{p} k\left(\lambda_{m} L\right)^{\beta_{m}}\left(r_{b}-r_{m}\right)-\left(\lambda_{m} L\right)^{\beta_{m}} r_{m}
\end{aligned}
$$

where, $S$ is total the number of product sold and $p$ is the proportion of product sold with such warranty and $q$ is the proportion sold without warranty. $a$ is the risk parameters representing the manufacturer's risk preferences. $k$ is the proportion of buyers without warranty, coming back to manufacturer for repairing of the failed products, $r_{m}$ and $r_{b}$ are the manufacturer's and buyer's per occasion repair cost the difference between the buyer's repair cost and manufacturer's per occasion repair cost, $\beta_{m}$ and $\lambda_{m}$ are the shape parameter and inverse characteristic life parameter for all products sold.

\section{SENSITIVITY ANALYSIS OF THE RISK MODELS}

In this section, we firstly present a sensitivity analysis of the buyer's intension to pay for warranty price with respect to the following factors: (1) buyer's risk preferences, (2) buyer's anticipated product failure intensity, and (3) buyer's repair costs, if not warranted. Secondly, we present analysis of the effect of manufacturer's optimal warranty price for (1) manufacturer's risk preference, (2) manufacturer anticipated product failure intensity, and (3) buyer's repair cost and return rate to the original manufacturer for repair if not warranted. 
1) Sensitivity Analysis of Buyer's Willingness to Pay for Warranty Price: for all cases let us assume that $60 \%$ of the sold products are with warranty and $40 \%$ products are sold without this type of warranty, which implies that $p=0.6$ and $q=0.4$ for all occasions of our analysis. For the purpose of simplicity, in this sensitivity analysis we assume the buyer's anticipated lifetime of the product $L=$ 3 years.

\section{Effect of buyer's risk preferences on the warranty price}

In this analysis part, the buyer's risk parameters ' $c$ ' is varied systematically from 0.1 to 1.0 representing a wide spectrum of buyer's risk preferences. A buyer become more risk averse as the risk parameter ' $c$ ' increases and he/she becomes risk neutral if the parameter is zero.

Let the buyer's anticipated non-homogeneous Poisson process parameters are: inverse characteristic life $\lambda_{\mathrm{b}}=$ $0.325 /$ year, and shape parameter $\beta_{\mathrm{b}}=2$, buyer's repair cost of each failure $r_{b}=\$ 30$, if not warranted and the buyer's repair cost is same for all occasions whether it is repaired by the manufacturer or by an outside repairer. A computer simulation program generates the Fig 1 for the buyer's acceptance of warranty price with variation of the buyer's risk preference parameter $c$ over the lifetime.

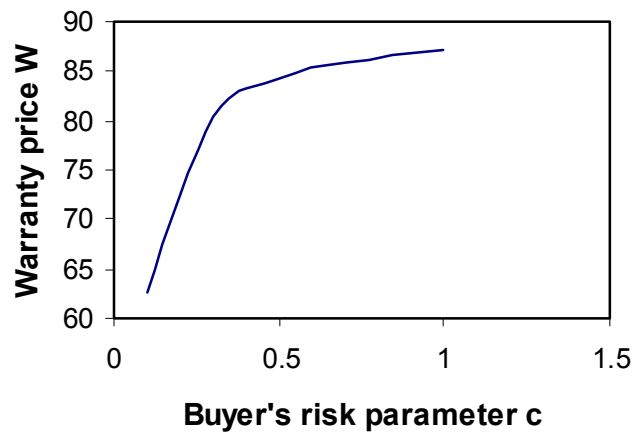

Fig 1: Effect of buyer's risk preference parameter on the warranty price when buyer anticipated product failure is $n=3$.

Fig 2 clearly states that the warranty price increases with the increase of the of the buyer's risk preference which indicates that, the warranty price increases as the buyer becomes more averse and the warranty price decreases as the buyer becomes less averse. This means that the buyer with higher risk averseness is willing to pay higher warranty price. Manufacturer or dealers can use this buyer's psychology while being pricing the warranty.

\section{Effect of buyer's repair cost on the warranty price}

Although the buyer's repair cost is applicable only for the buyers with non-warranted item, it has a significant effect on the warranty price. In this analysis, the buyer's rectification or repair cost for each failure is varied systematically from $\$ 30$ to $\$ 70$ representing a wide range of buyer's repair cost. It is noted that we assume this repair cost are constant for a particular product all along its lifetime.
We assume the buyer's anticipated non-homogeneous Poisson process parameters: inverse characteristic life $\lambda=$ $0.325 /$ year, and shape parameter $\beta=2$, buyer's risk parameter $c=0.5$. The computer program generates the Fig. 2 for the buyer's acceptance of warranty price with variation of the buyer's repair cost. The Fig 2 shows that the buyer's willingness for warranty price increases linearly as the buyer's repair cost increases. This implies that the buyers are ready to pay higher warranty price if the repair costs are higher. That is more proportion of buyers will be interested to buy warranty as the repair price goes up.

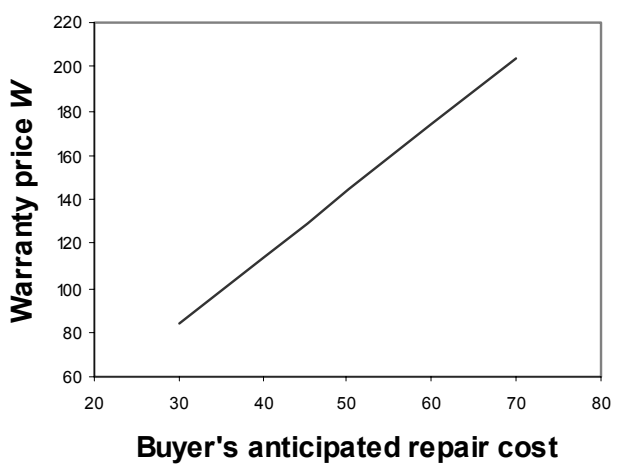

Fig. 2: Effect of buyer's repair cost on the warranty price

\section{Effect of buyer's anticipated product failure intensity on the warranty price}

Here, we analyse the sensitivity of buyer's anticipated product failure rate or intensity of failure on the buyer's acceptance of warranty price. To see the influence of product failure rate on the warranty price we vary the $\lambda_{b}$ from 0.125 to .525 . We assume the buyer's anticipated non-homogeneous Poisson process parameters shape parameter $\beta_{b}=2$, buyer's risk parameter $c=0.5$. The computer simulation program generates the Fig 3 for the buyer's accepted warranty prices with variation of the failure intensity parameter $\lambda_{b}$.

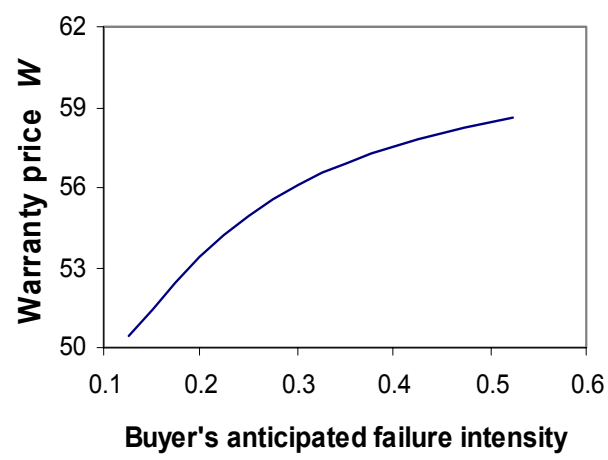

Fig. 3: Effect of buyer's anticipated failure intensity $\left(\lambda_{b}\right)$ on the warranty price.

The Fig. 3 shows that the buyer's willingness for warranty price increases as the buyer's anticipated failure intensity increases. This implies that the buyers are ready to pay 
higher warranty price for higher intensity of product failure. Conversely, when the buyers anticipate lower product failure intensity, they will be willing to pay less warranty price.

\section{2) Sensitivity Analysis of the Manufacture's Warranty Price}

In this subsection, we analyze the sensitivity of manufacturer's optimal warranty pricing with the variation of manufacturer's risk preference, manufacturer estimated product failure intensity, buyer's repair cost and buyer return rate to the manufacturer for repair of the failed product respectively.

\section{Effect of manufacturer's risk preference on the warranty price}

Similar to the sub-section 1), the manufacturer risk parameters ' $a$ ' is varied systematically from 0.05 to 1.0 representing a wide spectrum of manufacturer risk preferences. Let the manufacturer's estimated nonhomogeneous Poisson process parameters are: inverse characteristic life $\lambda_{m}=0.325 /$ year, and shape parameter $\beta_{m}$ $=2$, buyer's repair cost for each failure $r_{b}=\$ 30$ and manufacturer's actual cost of each repair $r_{m}=\$ 10$. Let the rate of returning of buyers $(\mathrm{k})$ to the manufacturer for repair of failed product is $20 \%$ that is $k=0.2$. A computer program generates the Fig. 4 for the manufacturer's optimal warranty price with variation of the manufacturer's risk parameter $a$. The Fig 4 shows that the warranty price increases with the increase of the of the manufacture risk preference parameter $a$. The warranty price increases as the manufacturer becomes more risk averse and the warranty price decreases as the manufacturer/dealer becomes less averse. This implies that the more a manufacturer is risk averse the more he/she will charge for the warranty.

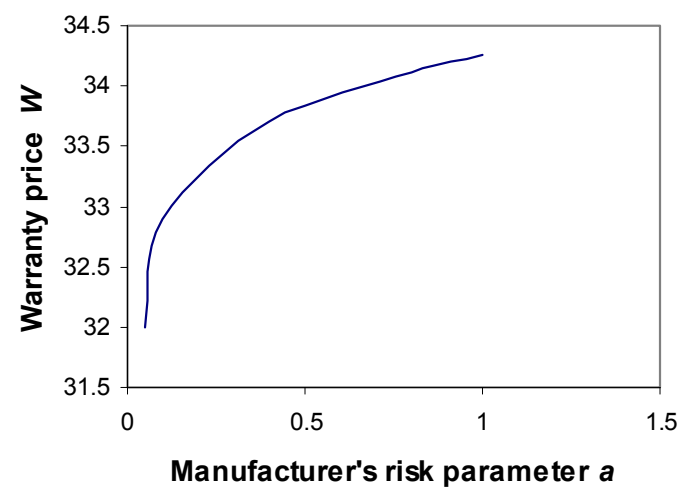

Fig. 4: Effect of manufacturer's risk parameter on the warranty price

\section{Effect of manufacturer's estimated product failure intensity on the warranty price}

In this analysis, we consider a range of manufacturer estimated inverse characteristic life $\left(\lambda_{m}\right)$ of product to represent failure intensity (rate) in observing the effect on the manufacturer's optimal charge for warranty. To do so, we vary $\lambda_{m}$ from a range of $0.125 /$ year to 0.443 . Keeping all other values as before, let the manufacturer risk parameters ' $a$ ' be 0.4 . The computer program generates the Fig 5 which exhibits the effect of failure intensity over the manufacture's charge for warranty price.

Analysis of Fig 5 shows that the manufacturer's charge for warranty price increases with the increase of the $\lambda_{m}$. This implies that, the higher the manufacturer estimates the failure intensity, the higher the manufacturer charge for the warranty price to meet the higher rate of failure consequently more warranty claims. Conversely, less failure intensity results in less warranty claims and the manufacturer is interested to offer less warranty price.

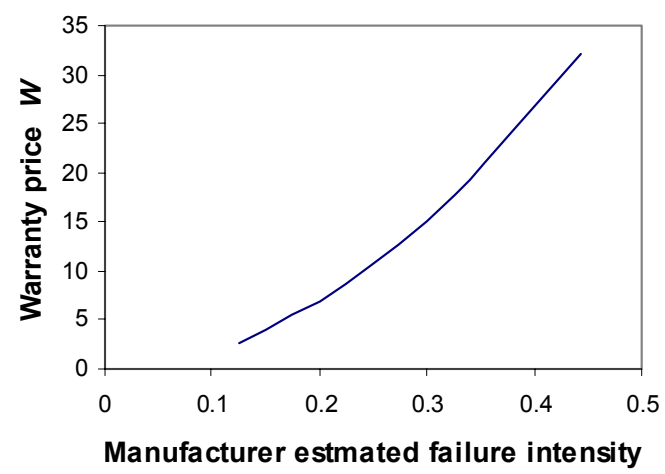

Fig. 5: Effect of manufacture's estimated failure intensity over warranty price.

\section{Effect buyer's repair costs on manufacturer warranty charge}

Now, we will look into the effect of buyer's repair costs on the manufacturer's charge for warranty price. Here, in this analysis, we vary the buyers repair costs for each occasion. Let buyer's repair cost $r_{b}$ be a range of $\$$ 40 to $\$ 80$ per occasion. We also assume that buyer's repair cost remain constant for each case. Values of all other variables and parameter are kept as before. The computer program generates the Fig 6 which shows the effect of buyer's cost of repair over the manufacture's charge for warranty price.

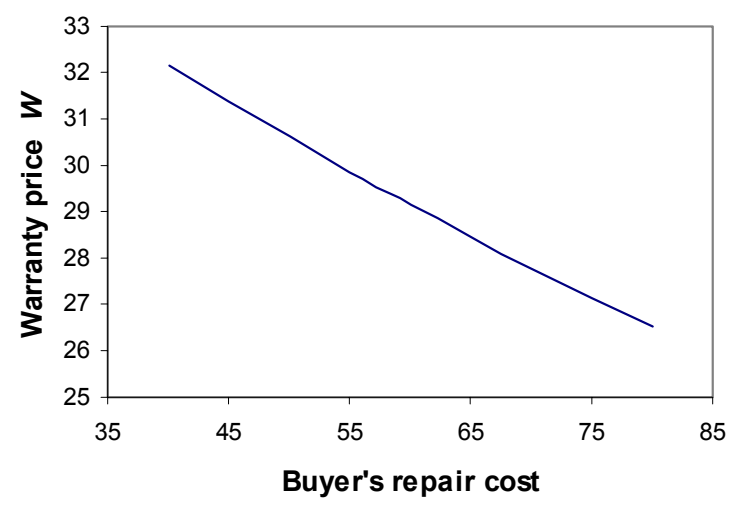

Fig. 6: Effect of buyer's cost of repair over the manufacture/dealer's charge for warranty price. 
Analysis of Fig 6 shows that the manufacturer's charge for warranty price decreases with the increase of the of the buyer's cost of repair. This occurs because when the buyer's repair cost is higher, the more buyer will prefer warranty and this in turns provides an opportunity for the manufacturer to lower the warranty price to bring more buyer within such warranty in order to compete effectively in the market.

\section{Effect of buyer rate of return (k) to the manufacturer for repair on the manufacturer charge for warranty price}

A portion of non-warranted buyer may come back to the original manufacturer for repair of their failed products. Here, we will analyze the effect of buyer rate of return $(k)$ to the manufacture for repair on the warranty price. For this purpose we vary the proportion of returning of buyers from $0 \%$ to $100 \%$, which implies different values of $k$ ranging from 0 to 1 . The computer program generates the Fig 7 which shows the effect of buyer's rate of return over the manufacture's charge for warranty price.

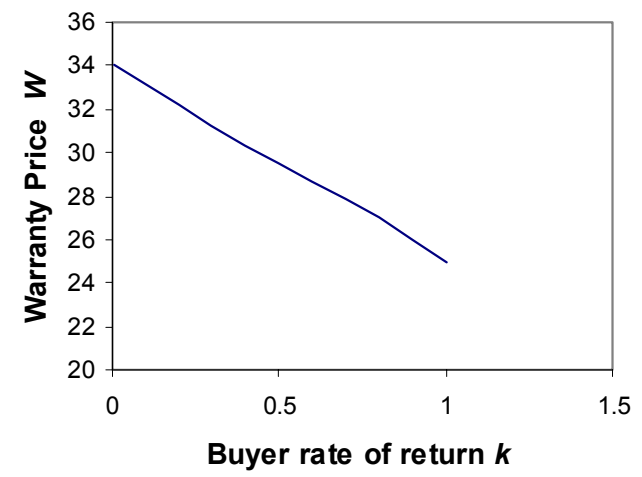

Fig.7: Effect of buyer's rate of return over the manufacture/dealer's charge for warranty price.

Analysis of Fig 7 shows that the manufacturer's charge for warranty price decreases with the increase of the rate of buyer's return to the original manufacturer/dealer for repair. This implies that the more the buyer come back to the manufacturer for repair, the more the manufacturer will encourage the buyers to buy the warranty.

\section{CONTRIBUTIONS AND FUTURE SCIOPES}

In this paper, sensitivity of the optimal warranty price is analyzed with respect to the manufacturer and buyer's risk attitudes towards a lifetime warranty policy for a product with time dependent failure mode. In doing so, sensitivities of risk models developed by Chattopadhyay and Rahman[1] were analyzed to see the effects of the buyer's and the manufacturer's risk preferences, buyer's anticipated and manufacturer's estimated product failure intensity, the buyer's loyalty to the original on the optimal warranty price. Numerical examples were used to demonstrate the application of this procedure to practical problems and highlight it's usefulness in a managerial context. Similar analysis can be carried out for risks preference models developed for products with time independent failure mode such as products with constant failure intensity for managerial decision in pricing both base and lifetime warranty.

\section{REFERENCES}

[1] G.N. Chattopadhyay, A. Rahman,. (2005) "Modelling Costs and Risks to Manufactures and Buyers for Lifetime Warranty Policies", Proceedings of the 18th International Congress on Condition Monitoring and Diagnostic Engineering Management, COMADEM 2004, Cranfield University, U.K. August - September, 2004. ISBN 1-871315-91-3. pp. 73-81.

[2] P.H. Ritchken and C.S. Tapiero, "Warranty design under buyer and seller risk aversion", Naval Research Logistics Quarterly, Vol. 33 pp.657-671, 1986.

[3] M.A.J. Menezes, "The Impact of Product warranties on consumer preferences", A draft paper submitted to Harvard Business School (Report No. 90-003), 1989

[4] Y.H. Chun and K. Tang, "Determining the Optimal Warranty Price Based on the Producer's and Customer's Risk Preferences." European Journal of Operational Research, Vol 85, pp 97-110, 1995.

[5] A. Rahman and G.N. Chattopadhyay, "Lifetime Warranty Policies: Complexities in Modelling and Potential for Industry Application", The Proceedings of the APIEM Dec 2004, Gold coast, Australia. ISBN 0-9596291-7-3, 2004.

[6] The Magnuson-Moss Warranty Act (1975) - United States federal law of warranties on consumer products, (15 U.S.C. $\$ 2301$ et seq.). Enacted in 1975, it is the federal statute that governs

[7] G.N. Chattopadhyay and A. Rahman, "Modelling Costs for Lifetime Warranty Policies", Proceedings of the $17^{\text {th }}$ International Congress on Condition Monitoring and Diagnostic Engineering Management, COMADEM 2004, Cambridge, U.K. August 2004, pp 289-297, 2004. 